\title{
TA-KONZEPTE UND -METHODEN
}

\section{Transdisziplinäre Umweltfor- schung als methodologische Aufgabe Reflexionen einer Forschungskoope-
ration von Biologie und Soziologie}

\author{
von Stefan Brunzel und Jens Jetzkowitz, \\ Universität Marburg
}

Ob transdisziplinäre Forschung, die allein auf die Veränderung von Strukturen der Wissenschaftsorganisation setzt, ihr Ziel erreicht, ist fraglich: Die von den Transdisziplinaritäts-Befürwortern angestrebten Veränderungen im Wissenschaftssystem scheinen die Probleme, die sie beseitigen sollen, noch zu verstärken. Innovatives Potenzial wird die fächerübergreifende Forschung nur dann entfalten, wenn sie sich auf eine methodologische Diskussion einlässt. Am Beispiel eines aktuellen Forschungsprojektes verdeutlichen wir eine Methodologie, mit der sich die Trennung zwischen der geisteswissenschaftlichen und der naturwissenschaftlichen Rationalität überwinden lässt.

\section{Einleitung}

In der Umweltforschung setzt sich mehr und mehr die Einsicht durch, dass die Naturgefährdungen, die von der modernen, industrialisierten Gesellschaft ausgehen, nicht allein durch naturwissenschaftlich-technische Rationalität $\mathrm{zu}$ beseitigen sind. Denn trotz der vielen Erfolge, die auf diesem Weg en détail erzielt wurden, sind die Erwartungen an grundlegende Veränderungen im Verhältnis von Mensch und Natur nicht erfüllt worden. Weder haben naturwissenschaftlich fundierte Mahnungen und Warnungen vor den Folgen der industriellen Produktionsweise und der Wachstumsideologie, die sie legitimiert, $\mathrm{zu}$ grundlegenden Änderungen gesellschaftlicher Strukturen geführt, noch haben technische Innovationen, ökonomische Anreize und rechtliche Restriktionen die anthropogen induzierten Umweltveränderungen in einer Weise korrigiert, dass man heute von nachhaltigen Verhaltens- weisen gegenüber der Natur in der modernen Gesellschaft sprechen könnte.

Wer Erwartungen enttäuscht, verliert meist das Vertrauen, und so wird heute verstärkt an Denktraditionen angeknüpft, die auf die Probleme der naturwissenschaftlich-technischen Rationalität aufmerksam machen. $\mathrm{Zu}$ erinnern ist beispielsweise an Husserls (1982) eindringliche Warnung vor der objektivistischen Verengung der neuzeitlichen Wissenschaft, die das Subjekt - und mit ihm auch die Fragen nach Sinn und Relevanz - aus der Forschung ausgeschlossen hat, ${ }^{1}$ oder an Horkheimers ,Kritik der instrumentellen Vernunft" (1967). ${ }^{2}$ Dank dieser und anderer Einflüsse wird in der Umweltforschung wieder über grundlegende Denk- und Forschungsvoraussetzungen und über Paradigmen nachgedacht. Leitend ist dabei der Gedanke, dass die Probleme, auf die sich die Umweltforschung bezieht, quer zur Aufteilung der Welt in Wissenschaftsdisziplinen liegen.

„Transdisziplinarität" lautet folglich das Schlagwort, an dem sich die Neuausrichtung der Umweltforschung orientiert. Die damit verbundenen Bemühungen können sich derzeit auf die breite Unterstützung deutscher Forschungsförderer verlassen, die vor allem den wissenschaftlichen Nachwuchs auffordern, disziplinenübergreifend $\mathrm{zu}$ arbeiten (Scheringer 2002; Hof 2002; Wächter 2002; Grunwald 2002). Diese Förderinitiativen regen dazu an, sich auf die Suche nach neuen Wegen in der Umweltforschung $\mathrm{zu}$ begeben und schlicht und einfach auszuprobieren, wie sich transdisziplinäre Forschung realisieren lässt. Im Rahmen unseres aktuellen Forschungsprojektes „Die Zusammenhänge zwischen den Veränderungen in der dörflichen Vegetation häufig gestörter Lebensräume und dem Wandel ländlicher Lebensstile“" bietet sich uns derzeit die Gelegenheit, fächerübergreifende Forschung aus den Perspektiven der Biologie und der Soziologie durchzuführen und zu reflektieren. ${ }^{3}$ In der gemeinsamen Arbeit ist uns dabei aufgefallen, dass sich die rege Debatte über Transdisziplinarität in der Umweltforschung vor allem auf die Veränderung von Organisationsstrukturen der Forschung konzentriert. Hingegen ist das methodologische Spannungsfeld, das bei der Zusammenarbeit von Natur- und Geisteswissenschaften entsteht, bislang kaum thematisiert worden. 
Mit den folgenden Ausführungen wollen wir einen Beitrag zu einer solchen Diskussion leisten. Dabei gehen wir davon aus, dass sich Innovationsblockaden in der Umweltforschung erst auflösen, wenn dieses Spannungsfeld überwunden wird und die Erforschung von Mensch-Natur-Verhältnissen jenseits disziplinärer Zwänge und Selbstverständlichkeiten ansetzt. Unsere Überlegungen zur Methodologie der fächerübergreifenden Umweltforschung sollen verdeutlichen, wie nach solchen Ansatzpunkten gesucht werden kann.

\section{Transdisziplinarität als organisatori- sches Problem?}

Aus soziologischer Perspektive betrachtet, stellt sich die Transdisziplinaritätsdebatte in der Umweltforschung als ein Konflikt dar, der in einer gewissen Unzufriedenheit mit den gegenwärtig herrschenden, etablierten Strukturen der Umweltforschung begründet liegt. Welche konkreten Ursachen die Unzufriedenheit im Einzelnen hat, ist sekundär, sobald sie in der Forderung nach einer anderen, eben: transdisziplinären Wissenschaft eine Sprachform gefunden hat, die verschiedene Motivationen bündeln kann. Der Begriff der Transdisziplinarität, der von Jürgen Mittelstraß (1992) in die Diskussion über die Umweltforschung eingebracht wurde (vgl. auch Jaeger und Scheringer 1998, S. 10 f.), kann dies offenbar leisten. Er bringt hinreichend allgemein zum Ausdruck, dass die Strukturen der etablierten Umweltforschung als, salopp gesprochen, verkrustet erlebt werden. Der Protest richtet sich dabei gegen die Organisation der Wissenschaft in Disziplinen, weil diese sowohl die Definition des Forschungsgegenstandes als auch die Methoden der Forschung beschränken.

In der Tat wirken sich die Wissenschaftsdisziplinen durch ihre spezifischen Verflechtungen von Wissens- und Machtstrukturen reglementierend auf das aus, was in der scientific community erforscht und kommuniziert werden kann. ${ }^{4}$ So ist jeder Wissenschaftler, der sich mit Umweltproblemen beschäftigt, daran gebunden, sich an den Fragestellungen und den Sprachgebräuchen seiner Wissenschaftsdisziplin zu orientieren, um am Fachdiskurs teilzunehmen und z. B. eine Zeitschrift für die Publikation seiner Forschungsergebnisse zu finden. Die verschiedenen sozialen Kontrollmechanismen, die ihm in Form von Gutachtergremien bei der Beantragung von Forschungsgeldern, von referees bei der Einreichung von Aufsätzen bei einer Zeitschrift oder von Berufungskommissionen bei einer Bewerbung um eine Professur entgegentreten, sichern die Diskussions- und Argumentationsstandards einer Wissenschaft. Solche Instanzen sondern aus, was nicht als Beitrag zur Erforschung des spezifischen Gegenstandes der Disziplin gerechnet wird oder was methodisch fragwürdig ist. Diese Praxis ist durchaus ambivalent, denn sie verhindert zugleich auch, dass in einem Diskurs grundlegende Irritationen entstehen und die Diskussions- und Argumentationsstandards neu justiert werden müssen. Darum können sich die Diskussions- und Argumentationsstandards auch als Innovationsblokkaden auswirken.

Was aber kann ein Wissenschaftler tun, wenn er für seine Ideen kein Gehör findet? Wer sich aus dem Wissenschaftsdiskurs nicht enttäuscht zurückziehen will, dem bleibt die Möglichkeit, Protest in der Sprache der Wissenschaft $\mathrm{zu}$ artikulieren und z. B. eine Metadiskussion über die Strukturen der wissenschaftlichen Forschung zu eröffnen. Die Transdisziplinaritätsdebatte in der Umweltforschung ist eine solche Metadiskussion, in der sich Protest gegen die etablierte disziplinäre Struktur der Wissenschaft äußert. Wie in Protestbewegungen anderer Lebensbereiche, konkretisieren die Befürworter transdisziplinärer Forschung ihre Forderungen mit durchaus verschiedenen Programmen (vgl. z. B. Jaeger und Scheringer 1998; Bergmann 2003, S. 65-67). ${ }^{5}$ Gemeinsam ist ihnen aber die Wahrnehmung, dass sich der professionelle Wissenschaftsbetrieb gegenüber der gesellschaftlichen Lebenswelt entfremdet hat: Die Wissenslandschaft habe sich in einem solchen Ausmaß in kleine, hochgradig spezialisierte Diskurse fragmentiert, dass sowohl die Kommunikation zwischen den verschiedenen Forschungsbereichen als auch die zwischen Wissenschaft und gesellschaftlicher Praxis grundlegend blockiert seien. Um diese Blockade zu überwinden und die Problemlösungskompetenz der Wissenschaft wiederherzustellen, muss - so die Befürworter der Transdisziplinarität - die Forschung dort ansetzen, wo die Probleme liegen: jenseits ihrer Interpretation in den Disziplinen der Wissenschaft. Die Arbeit am Transdisziplinaritätskonzept ist also, so lässt sich resü 
mieren, mit der Vision von einer Wissenschaft verbunden, die Probleme gesellschaftlicher Praxis löst, ohne sich dabei zu verselbstständigen und die Frage nach der Relevanz der Forschung aus dem Blick zu verlieren.

$\mathrm{Ob}$ diese Vision aber allein durch die Etablierung neuer, transdisziplinärer Forschungseinrichtungen und Studiengänge wirksam wird, muss nach dem gegenwärtigen Stand der Transdisziplinaritätsdebatte bezweifelt werden. Denn zum einen findet auch transdisziplinäre Forschung unter den Bedingungen statt, die für Wissenschaft allgemein gelten. Sie muss die Nachvollziehbarkeit und die Kritisierbarkeit ihrer Ergebnisse sicherstellen und dafür Forschungs- und Argumentationsstandards ausbilden. Sie kann also immer ,nur" wissenschaftliche Forschung sein und hat nicht per se eine größere Nähe zur gesellschaftlichen Praxis als disziplinäre Forschung (Büchi 1996, S. 205). Zum anderen hebt die Etablierung transdisziplinärer Forschung nicht die disziplinären Spezialisierungen in der Wissenschaft auf, sondern verstärkt sie vielmehr dadurch, dass neue Wissensund Fachgebiete erschlossen und womöglich auch neue Studiengänge eingerichtet werden (vgl. Weingart 1997). Aus diesem Blickwinkel betrachtet führt die Forderung und Förderung von transdisziplinärer Forschung also in eine Aporie. Denn es scheint so zu sein, dass die Charakteristika der etablierten, disziplinären Wissenschaft, gegen die sich die Transdisziplinaritäts-Befürworter wenden, im Falle ihres Erfolges nicht verschwinden, sondern sich sogar noch verstärken werden.

\section{Transdisziplinäre Umweltforschung als erfahrungswissenschaftliche Naturphilo- sophie}

Die Entzauberung des Transdisziplinaritätskonzepts durch seine Kritiker lässt die Forderung und Förderung von transdisziplinärer Umweltforschung in einem anderen Licht erscheinen. Sie leitet über zu der Überlegung, ob die Innovationsblockaden in der Umweltforschung Folgen einer spezifischen Denkgewohnheit sind, die uns das Natur-Mensch-Gefüge entweder aus der Perspektive der Naturwissenschaften oder aus der der Geisteswissenschaften betrachtet lässt: Wir sind es gewohnt, die Ereignisse in der Welt zu ordnen, indem wir strikt zwischen Natur und Geist - alternativ: zwischen Natur und Kultur - als den beiden grundlegenden Bereichen des Wirklichen unterscheiden (vgl. dazu auch Balsinger 1996).

Zwar ist diese Denkgewohntheit, die Rene Descartes seiner dualistischen Naturphilosophie erstmals systematisch ausgearbeitet hat, nur eine unter mehreren Möglichkeiten, um die Welt zu verstehen und die Wissenschaften zu klassifizieren. In der okzidentalen Kulturgeschichte hat sie sich aber als besonders einflussreiche Tradition etabliert, die es geradezu notwendig erscheinen lässt, wissenschaftliche Rationalität in zwei verschiedenen Schemata auszuformen. In Diltheys Diktum „Die Natur erklären wir, das Seelenleben verstehen wir" (Dilthey 1924, S. 144) findet das pointiert Ausdruck. Diese beiden Schemata haben sich zu zwei verschiedenen Wissenschaftskulturen verselbstständigt, deren Wirksamkeit Charles Percy Snow 1959 unter dem Titel „The Two Cultures" auf anschauliche Weise vorgeführt hat (Snow 2001). Er beschreibt einerseits eine naturwissenschaftlichtechnische Kultur und andererseits eine geisteswissenschaftlich-literarische Kultur, die beide intern durch Spezialisierung und in ihren Außenverhältnissen durch Ignoranz gegenüber der anderen Seite gekennzeichnet sind. Das gegenseitige Nichtverstehen, das sogar mit einer gewissen Feindseligkeit einhergeht, wirkt sich, Snows Diagnose zufolge, unheilvoll auf das geistige Leben aus, weil es das Problemlösungspotenzial der Wissenschaft blockiert.

Nirgends sind diese Blockaden offensichtlicher als in der Umweltforschung. Denn dieser Forschungsbereich ist ja entstanden, um gerade die anthropogen induzierten Naturveränderungen zu untersuchen und Entwicklungsoptionen für die menschliche Gesellschaft aufzuzeigen, die die Zerstörung ihrer Voraussetzungen vermeidet. Folglich liegt es nahe, die Forschungen jenseits der Unterscheidung von Natur- und Geisteswissenschaften anzusetzen und gerade nach den Wechselbeziehungen von Natur und Kultur zu fragen. Die Rufe nach Transdisziplinarität scheinen daher in der Umweltforschung besonders plausibel zu sein. Die Schwierigkeiten zeigen sich dann aber, wenn bei der Konzipierung und Durchführung transdisziplinärer Umweltforschung die Frage auftaucht, wie sich die unterschiedlichen methodischen Zugänge der verschiedenen Wissenschaftskulturen zum 
Natur-Mensch-Gefüge sinnvoll aufeinander beziehen lassen. ${ }^{6}$

In diesem Kontext ist der Verweis auf bereits bestehende Traditionen transdisziplinären Denkens hilfreich, die sich im philosophischen Diskurs gebildet haben, aber auch auf Systemforschung und Humanökologie (Tretter 1997). Solche Traditionen machen darauf aufmerksam, dass in der Wissenschaftsgeschichte immer wieder intellektuelle Bewegungen die Einheit der wissenschaftlichen Rationalität hervorgehoben haben. Sie haben zwar das Methodenproblem nicht gelöst, aber die Möglichkeit von Alternativen zur cartesianischen Naturphilosophie aufgezeigt. Für die transdisziplinäre Umweltforschung sollte dies u. E. Anregung sein, sich von der Verpflichtung auf eine einzige Naturphilosophie zu lösen, die - häufig implizit die Auseinandersetzung mit dem Forschungsfeld strukturiert. Dies gilt in besonderem Maße für die Naturwissenschaften, die noch stärker als die Geisteswissenschaften die Annahme voraussetzen, dass Natur und Geist, Physisches und Mentales, kategoriale Gegensätze sind, und sie mit der Vorstellung verbinden, dass die Natur durch den menschlichen Geist, der die naturgegebenen Zusammenhänge analysieren und in seinem Sinne beeinflussen kann, beherrschbar ist. Da es ja gerade die durch diese Naturphilosophie begründete Handlungslogik ist, die den problematischen Umgang unserer Zivilisation mit der Natur hervorgebracht hat, sollte sie zumindest nicht als einzig mögliche Form betrachtet werden, um das Verhältnis von Natur und Kultur zu denken.

Die Erforschung des Natur-Mensch-Gefüges kann aber auf solche naturpilosophischen Festlegungen verzichten. Sie kann bei der trivialen Einsicht ansetzen, dass menschliches Handeln die Natur verändert und dass diese Veränderungen als regelhafte Zusammenhänge erfahrbar sind und systematisch erforscht werden können. Das heißt aber nicht, gänzlich auf naturphilosophische Spekulationen zu verzichten. Im Gegenteil, es ist geradezu die vernachlässigte Aufgabe der Umweltforschung, ihre naturphilosophischen Implikationen zu explizieren und zur Diskussion zu stellen. Denn nur auf diese Weise bietet sich der transdisziplinären Umweltforschung die Chance, die wissenschaftlichen Weltbilder, auf denen sie aufbaut, selbst zu korrigieren. So sind es ja gerade die überraschenden Beobachtungen im NaturMensch-Gefüge, die in der Forschungspraxis zu neuen Einsichten führen. Überraschend ist eine Beobachtung aber immer nur vor dem Hintergrund einer Erwartung, die skizziert, welche Beobachtungen man eigentlich machen müsste, wenn die Welt so funktionieren würde, wie man annimmt, dass sie es täte. Dass dieser Erwartungshintergrund aber in jedem Fall eine metaphysische bzw. naturphilosophische Dimension hat - dass jeder Forschungsprozess immer auch auf Voraussetzungen aufbaut, die nicht durch Erfahrungen gestützt sind -, wird heute kaum noch bestritten. Indem ein Erwartungshintergrund als eine Abduktion betrachtet wird, die im Forschungsprozess kritisch zu überprüfen ist, kann die transdisziplinäre Umweltforschung verschiedene Konzeptionen des Natur-MenschGefüges berücksichtigen und in einen rekursiven Lernprozess eintreten. ${ }^{7}$

\section{Wie die Sprachlosigkeit zwischen den beiden Wissenschaftskulturen überwun- den werden kann}

Das Konzept von transdisziplinärer Umweltforschung als einer erfahrungswissenschaftlichen Naturphilosophie erleichtert es anzuerkennen, dass sowohl die Natur- als auch die Geisteswissenschaften Methoden entwickelt haben, um die Verflechtungen von menschlichem Handeln und nicht-menschlicher Umwelt $\mathrm{zu}$ erforschen. Diese Anerkenntnis ist freilich erst der Ausgangspunkt, um die Sprachlosigkeit zwischen den Wissenschaftskulturen zu überwinden. Sie setzt Wissenschaftler mit verschiedenen fachkulturellen Sozialisationen dem sanften Druck aus, zu den Forschungsstrategien und -ergebnissen aus der jeweils anderen Wissenschaftskultur Stellung zu beziehen und sie als Beiträge zur Umweltforschung wahrzunehmen. Damit rückt die Frage in den Vordergrund, wie die verschiedenen methodischen Zugänge zum Natur-Mensch-Gefüge miteinander in Beziehung gesetzt werden können. Wie kann in der Auseinandersetzung mit einem konkreten Forschungsgegenstand eine gemeinsame Sprache entwickelt werden, die einerseits das Eigentümliche der jeweiligen disziplinären Perspektive zum Ausdruck bringt und andererseits die transdisziplinär relevanten Erkenntnis 
se über den gemeinsamen Forschungsgegenstand verständlich vermitteln kann?

In unserem Forschungsprojekt „Die $\mathrm{Zu}-$ sammenhänge zwischen den Veränderungen in der dörflichen Vegetation häufig gestörter Lebensräume und dem Wandel ländlicher Lebensstile" mussten wir auf diese Frage eine - wenn auch zunächst vorläufige - Antwort finden, um eine Verständigungsgrundlage für die gemeinsame Arbeit zu schaffen. Dabei hat sich bislang das Paradigma des Spurenlesens als ein geeigneter methodologischer Bezugsrahmen erwiesen, das u. a. von Carlo Ginzburg (1979), Gerhard Hard (1995) und von Umberto Eco und Thomas A. Sebeok (1983) vertreten wird. ${ }^{8}$ In der Tradition der Semiotik betrachten wir dabei eine Spur als eine bestimmte Zeichenform: Sie weist ihren Betrachter direkt auf das Objekt hin, durch das sie hervorgerufen wurde. Ein Feuer erzeugt Rauch, ein Pferd hinterlässt eine charakteristische Hufspur und ein Einbrecher womöglich seinen Fingerabdruck. Zeichen wie z. B. eine Rauchfahne oder ein Fingerabdruck sind Indizien (vgl. Peirce 1983, S. 65-66). Sie sind keine Produkte der Phantasie, sondern existieren ebenso, wie die von ihnen indizierten Objekte. Ohne Feuer kein Rauch und ohne Pferd keine Hufspur. Nichtsdestotrotz wird etwas nur dadurch zu einem Indiz, dass es so aufgefasst wird. Wer die Hufspur nicht erkennt bzw. nur eine Unebenheit im Lehmboden sieht, der wird nicht darauf schließen, dass sich an dem betreffenden Ort ein Pferd aufgehalten hat. Solche Erkenntnisprozesse sind natürlich irrtumsanfällig. Die Spur im Lehmboden könnte ja auch von einem Rind oder einem Hirsch hervorgerufen sein. Daher ist jedes Indiz kritisch daraufhin zu prüfen, auf welche verursachenden Tatbestände es verweist. Lässt sich eine allgemeine Regel formulieren, die angibt, unter welchen Bedingungen das indizierte Ereignis immer wieder auftritt? Entlang dieser Leitfrage suchen wir nach Indizien für eine konkret existierende Relation im Natur-Mensch-Gefüge und überprüfen, ob die von uns angenommene Relation tatsächlich existiert oder ob sie ein Phantasieprodukt ist.

So baut unser Forschungsprojekt auf der Beobachtung auf, dass Ortschaften im ländlich geprägten Raum noch einen höheren Anteil von Arten der ehemals typischen Dorfruderalflora aufweisen als Ortschaften in der Nähe von Bal- lungsräumen. Mit dem Begriff der „Dorfruderalflora" wird dabei eine ganze Reihe verschiedener Pflanzen - einheimische Arten und so genannte Alteinwanderer (Archaephyten) - zusammengefasst, die bevorzugt auf vom Menschen stark beeinflussten und häufig gestörten Standorten in Siedlungen vorkommen und bis in die erste Hälfte des 20. Jahrhunderts typisch für dörfliche Siedlungsbereiche waren. In der ökologisch-biologischen Forschung wird als Ursache dieses Phänomens auf die Vernichtung der von diesen Pflanzen benötigten Standorte sowie auf die Unterbrechung der Ausbreitungswege hingewiesen. Hiermit werden bereits anthropogen induzierte Umweltveränderungen als Erklärung des Rückgangs dieser „klassischen“ Ruderalflora herangezogen. Gleichzeitig breiten sich aber z. B. Neophyten - eingewanderte Pflanzen, die nicht zur ursprünglich heimischen Flora gehören - vor allem in Siedlungsbereichen aus. Sie besiedeln dabei ruderale Standorte, die es offenkundig auch heute noch gibt, auf denen aber Vertreter einer „klassischen“ Ruderalflora kaum noch zu finden sind.

Man kann diese beiden Beobachtungen isoliert nebeneinander stehen lassen. Man kann sich aber auch durch ihre scheinbare Widersprüchlichkeit irritieren und dazu anregen lassen, eine weiterführende Erklärung für die Phänomene zu finden. Diese müsste u. E., auf Grund der Vorstellung von einem NaturMensch-Gefüge, das menschliche Handeln stärker als Erklärungsfaktor berücksichtigen. Ist vielleicht der Wandel der gesellschaftlichen Strukturen die verursachende Kraft, welche die biologischen Phänomene erklären könnte? Orientieren sich Pflanzen gar an den Wohnquartiersbewertungen von Menschen? In der Tat lassen sich ja seit den 1950er Jahren Veränderungen in der Sozialstruktur ländlicher Siedlungsbereiche konstatieren, die mit den Schlagworten der "Entagrarisierung" und der zunehmenden „Verstädterung" beschrieben werden (vgl. z. B. Henkel 1999, S. 90-91). Wenn die Verbreitung von „klassischen“ Dorfruderalarten und Neophyten auch durch gesellschaftlich erzeugte Tatbestände induziert ist, dann könnten die Beobachtungen erklärt werden. Mit dieser Hypothese, die von einem Zusammenhang zwischen dem Wandel ruderaler Artensets und dem Wandel ländlicher Lebensstile ausgeht, ist aus den zunächst iso 
liert betrachteten Phänomenen eine Spur geworden. Anhand von Beispielen aus unserem Untersuchungsgebiet lässt sich verdeutlichen, wie durch diese Hypothese Zeichen als Indizien für ein verursachendes Objekt lesbar werden.

Unser Untersuchungsgebiet ist die Wetter$\mathrm{au}$, eine alte Kulturlandschaft, die sich nördlich von Frankfurt erstreckt, im Westen durch den Taunus und im Osten durch den Vogelsberg und seine Ausläufer beschränkt wird. Als eine Region mit einer traditionell landwirtschaftlichen Prägung hat dieses Siedlungsgebiet seit den 1950er Jahren verschiedene Veränderungen erfahren. $\mathrm{Zu}$ nennen sind hier vor allem der Strukturwandel in der Landwirtschaft, aber natürlich auch der Ausbau von Frankfurt am Main als Dienstleistungszentrum, die Ansiedlung von produzierendem Gewerbe an verkehrsgünstigen Standorten in der Region und schließlich der Ausbau von Mittelzentren, die für die politische Verwaltung und den Handel bedeutsam sind (Friedberg, Bad Nauheim, Butzbach, etc.).

Unsere Untersuchungen können auf Daten einer Ruderalflorakartierung in dieser Region zurückgreifen, die Dr. Wolfgang Ludwig in dieser Region von 1974 bis 1981 durchgeführt hat. Vergleicht man diese Daten mit den Ergebnissen der aktuellen Ruderalflorakartierung, die wir 2002 im Rahmen unseres Projektes durchgeführt haben, bestätigt sich für die Ortschaften der Wetterau ein Trend, der in der einschlägigen Literatur zur „klassischen“ Dorfruderalflora beschrieben ist (Dechent 1988; Otte und Ludwig 1990; Brandes und Griese 1991; Lienenbecker und Raabe 1993): Viele Bestände der ehemals für Dörfer typische Ruderalarten wie etwa Guter Heinrich (Chenopodium bonus-henricus), Herzgespann (Leonurus cardiaca) und Bilsenkraut (Hyoscamus niger) sind durch einen drastischen Rückgang gekennzeichnet. Eine Ausnahme bildet aber z. B. die Eselsdistel (Onopordum acanthium), die ebenfalls als ,klassische“ Dorfruderalart gilt. Sie ist entgegen dem in der Forschungsliteratur konstatierten Trend in unserem Untersuchungsgebiet nicht seltener, sondern offensichtlich sogar etwas häufiger geworden ist. Warum? Da sich dieses Phänomen nicht ohne weiteres mit ökologischen oder standortkundlichen Interpretationen erklären lässt, bleibt in einer disziplinär beschränkten biologischen Perspektive nur die Möglichkeit, die Verbrei- tung der Eselsdistel als „Ausreißer“ aus dem allgemeinen Trend hinzunehmen.

Im Kontext unserer Hypothese wird dieses Phänomen zu einer Spur, die auf ein Handlungsmuster als verursachendes Objekt verweist. So haben wir bei unserer Ruderalflorakartierung die Eselsdistel einige Male - sowohl als Jungpflanze als auch als blühendes Exemplar - in ansonsten völlig von „Unkraut“ befreiten Gartenbeeten vorgefunden. Diese Beobachtung legt die Vermutung nahe, dass diese Art zuweilen bewusst von Unkrautbekämpfungsmaßnahmen ausgespart wird, und zwar weil ihr die Gartennutzer aufgrund ihrer imposanten Größe oder ihrer attraktiven roten Blüten einen besonderen ästhetischen Wert zuschreiben. Das bedeutet aber nichts anderes, als dass kulturelle Faktoren einen direkten Einfluss auf die Zusammensetzung von Pflanzengemeinschaften und deren Entwicklung ausüben. Eigentlich nicht verwunderlich, wenn man bedenkt, dass Menschen durch Jahrtausende währende Nutzung in der Kulturlandschaft bestimmte Pflanzengemeinschaften selektiert haben, wenn auch vielleicht weniger deutlich an der Umsetzung ästhetischer Ziele orientiert als es die konkrete Hypothese über die Schonung der Eselsdistel nahelegt.

Unser zweites Beispiel greift darum auch eine Spur auf, die auf nicht-intendierte Folgen eines Handlungsmusters verweist: Der als Neophyt eingestufte Australische Gänsefuß (Chenopodium pumilio) war Ende der 70er bzw. Anfang der 80er Jahre zwar im Zentrum von Frankfurt zu finden (vgl. Ludwig 1972; Ludwig, unveröffentl. Daten), ein Vorkommen in den Wetterauer Ortschaften konnte aber - wie unsere historischen Daten zeigen - nicht nachgewiesen werden. Während unserer ersten Ruderalflorakartierung konnten wir nun Chenopodium pumilio in einem Ort des Untersuchungsgebietes nachweisen, und zwar an der Endhaltestelle einer Buslinie, die diesen Ort mit der U-Bahnstation Nord-Westzentrum in Frankfurt verbindet. Im Kontext unserer transdisziplinär ausgerichteten Hypothese erscheint diese Beobachtung als Indiz für ein Handlungsmuster, das diese Ausbreitung von Chenopodium pumilio verursacht haben könnte. Denkbar ist, dass jemand in Frankfurt in unmittelbarer Nähe zu einer fruchtenden Pflanze von Chenopodium pumilio in Hundekot getreten ist. Im Bus konnte der Schuh nicht gereinigt werden, aber sofort 
nach dem Aussteigen an der End-Haltestelle ist der Hundekot an der Kante des Pflasters der Bushaltestelle abgestreift worden. Unabhängig davon, ob sich im konkreten Einzelfall die Ausbreitung genau so abgespielt hat, lässt sich darauf schließen, dass Pendler als Ausbreitungsmedium fungieren können. In Ortschaften, in denen viele Menschen ihren Arbeitsplatz in Frankfurt haben, wäre demgemäß die Ausbreitung von Chenopodium pumilio wahrscheinlicher als in Ortschaften mit einer geringen Pendlerzahl.

Diesen hier beispielhaft skizzierten Indizien, die möglicherweise auf konkrete Zusammenhänge im Natur-Mensch-Gefüge verweisen, gehen wir in unserem Forschungsprojekt nach. Wir können derzeit noch nicht davon berichten, ob sich die hypothetisch angenommenen $\mathrm{Zu}-$ sammenhänge als zutreffend erweisen bzw. ob wir Regeln finden, welche die von uns als Indizien betrachteten Phänomene erklären, oder ob sie zufällig entstanden sind. Die Auswertung der historischen Daten, die von 1974 bis 1981 erhoben wurden, hat aber bereits Hinweise ergeben, die zu Zweifeln an rein geoökologischen Erklärungen führen. Berechnet man beispielsweise für 60 der für die Untersuchung ausgewählten Ortschaften die Anzahl der Neophyten als Anteil an der vorgefundenen Ruderalflora und setzt sie in ein Verhältnis zu der Entfernung der Ort- schaften nach Frankfurt am Main, so ergibt sich folgender hochsignifikanter Zusammenhang (vgl. Abb. 1): Je weiter ein Ort von Frankfurt entfernt ist, desto geringer ist der Anteil der Neophyten an der im jeweiligen Ort festgestellten Ruderalflora.

Dieser statistisch abgesicherte Zusammenhang löst sich jedoch auf, wenn weitere sieben Ortschaften unseres Samples hinzugenommen werden, die in der Nähe Hungens im Norden der Wetterau liegen. Diese Orte weisen einen relativ hohen Neophytenanteil auf, obwohl sie zu denjenigen mit der größten Entfernung nach Frankfurt gehören, und weichen damit von der Regel ab. Da diese Orte ähnlich groß sind wie andere, in enger Nachbarschaft zu Orten liegen, die sich gemäß des Zusammenhangs verhalten, sich im selben Naturraum befinden, auf gleichen Böden liegen etc., lässt sich dieses Phänomen im Rahmen rein fachdisziplinär ökologischer, inklusive geographischer oder geologischer, Erklärungsansätze kaum plausibel machen. Im Rahmen unserer Hypothese, die von einem - partiell von geoökologischen Faktoren entkoppelten - Zusammenhang zwischen dem Wandel ruderaler Artensets und dem Wandel ländlicher Lebensstile ausgeht, könnte das Phänomen jedoch möglicherweise erklärt werden.

Entscheidend für die transdisziplinäre Umweltforschung scheint uns zu sein, dass wir

\section{Abb. 1: Zusammenhang zwischen dem Anteil der Neophyten und der Entfernung zu Frankfurt a.M.}

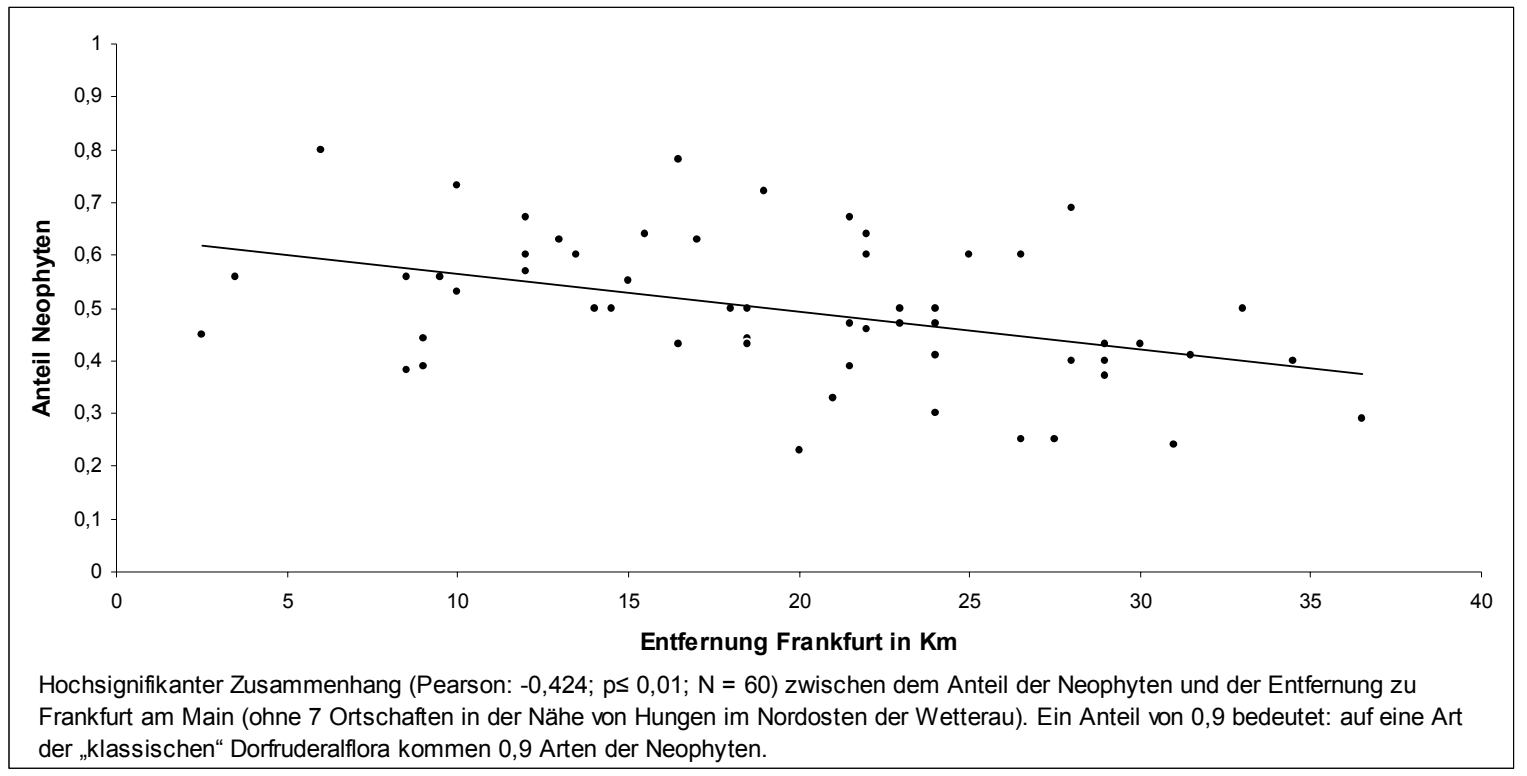


von einem methodologischen Bezugsrahmen ausgehen, der für verschiedene naturphilosophische Konzeptionen offen ist. Unsere Lesart des Spurenleser-Paradigmas legt es dabei nahe, die einzelnen Indizien, die auf konkrete NaturMensch-Interaktionen verweisen, in eine verallgemeinerte Regel zu überführen, welche Voraussagen über den Einfluss von Lebensstilaspekten auf die Ruderalflora ermöglicht. Nur wenn die Konsequenzen von Handlungsmustern für diese Biozönosen unabhängig vom Einzelfall überprüft werden, kann unsere Untersuchung zu einer Erklärung führen, die auch außerhalb des einzelnen Falles verwendet werden kann. Indem wir die Daten gemäß den disziplinären Methodenstandards erheben, vermeiden wir Kurzschlüsse im Sinne einer self-fulfilling prophecy. Das heißt, zum Zeitpunkt der Erhebung wissen wir nicht, ob sie unsere Hypothesen stützen oder widerlegen werden. Erst die Auswertung der Daten wird zeigen, ob sich die beobachteten Phänomene auf Ebene des Physischen erklären lassen und der Lebensstilwandel irrelevant ist oder ob sich die Indizien bestätigen.

\section{Schlussbemerkungen}

Transdisziplinäre Umweltforschung ist keine Wissenschaftspraxis, die sich formal-organisatorisch herstellen lässt, sondern sie benötigt vor allem inhaltliche Bezugspunkte. Diese entstehen, so unsere Überlegung, wenn phänomenbezogen über mögliche Vernetzungen von Mensch und Natur spekuliert wird und diese Spekulationen kritisch und erfahrungswissenschaftlich überprüft werden. Verbunden mit dem Zwang, gemeinsame Erfahrungen mit einem konkreten Forschungsgegenstand $\mathrm{zu}$ deuten, kann eine Sprache entstehen, die das Eigentümliche der jeweiligen disziplinären Perspektive zum Ausdruck bringt und zugleich auch die Einsichten über einen Forschungsgegenstand vermittelt, die von fächerübergreifendem Interesse sind.

Die Suche nach einer solchen Sprache ist ein zeitintensiver Lernprozess, der sich wohl kaum ohne Verständnisschwierigkeiten entwickeln wird. Angesichts dieser Schwierigkeiten kann die Orientierung an vermeintlich allgemeinverständlichem Wissen ebenso wenig als Königsweg angesehen werden wie die Verwendung von Alltagssprache bzw. der Verzicht auf fachsprachliche Terminologie. Denn die Bildungs- und Sprachcodes können sehr unterschiedlich sein. Unseres Erachtens ist bei der Suche nach einer solchen Sprache unabdingbar, dass Forscher aus verschiedenen Wissenschaftskulturen in der Auseinandersetzung mit einem gemeinsamen Forschungsgegenstand miteinander Erfahrungen sammeln und einander kennen lernen.

\section{Anmerkungen}

1) In seiner letzten und unvollendet gebliebenen Schrift „Die Krisis der europäischen Wissenschaften und die transzendentale Phänomenologie" diagnostiziert Husserl das Versagen der europäischen, rationalen Kultur. Die Eliminierung des Subjekts aus dem Bereich der Forschung habe Fragen gleichgültig beiseite geschoben, in denen es um Sinn oder Sinnlosigkeit des menschlichen Daseins, um Vernunft oder Unvernunft des Menschen, seine Freiheit und Verantwortlichkeit geht. Auf diese Weise sei den Wissenschaften ihr Sinnfundament verloren gegangen: die Lebenswelt.

2) In „Kritik der instrumentellen Vernunft“ macht Horkheimer deutlich, wie die Durchsetzung universeller Vernunftprinzipien systematisch zur Steigerung der Kontrolle über das Subjekt, über gesellschaftliche Verhältnisse und auch über die Natur führt.

3) Dieses Forschungsprojekt wird von der Volkswagen-Stiftung im Rahmen der ,Nachwuchsförderung in der fächerübergreifenden Umweltforschung" seit Februar 2002 gefördert.

4) Dass Wissen und Macht in einem engen symbiotischen Verhältnis stehen, ist geradezu sprichwörtlich. In der soziologischen Forschung ist dies in besonderer Klarheit vor allem von Michel Foucault (1980) herausgearbeitet worden.

5) So gehen beispielsweise Gibbons et. al. (1994, S. 3-44) deskriptiv vor und beschreiben einen in Entstehung befindlichen neuen Wissenschaftstyp, bei dem Wissen in flexiblen Organisationsstrukturen und anwendungsbezogen bei entsprechenden opportunistischen Interessenlagen produziert wird. Jaeger und Scheringer argumentieren dagegen stärker normativ und fordern transdisziplinäre Forschung als eine Ergänzung zu disziplinärer, multidisziplinärer und interdisziplinärer Forschung, um fächerübergreifende Probleme mit wissenschaftsexterner Wurzel zu bearbeiten.

6) Zu Recht hat Grunwald (1999) in der Transdisziplinaritätsdebatte darauf hingewiesen, dass ein Abbau oder gar ein Verzicht auf methodische Standards in der transdisziplinären Umweltfor 
schung kein gangbarer Weg ist. Ohne methodische Reflexion kann sich wissenschaftliche Forschung über das Zustandekommen ihrer Urteile keine Rechenschaft geben. Sie würde die Fähigkeit zur Selbstkorrektur verlieren, die sie, Peirce (1985) zufolge, von anderen Formen der Überzeugungsfindung unterscheidet.

7) Den Abduktionsbegriff erläutert Charles S. Peirce (1966) folgendermaßen: „Abduction is the process of forming an explanatory hypothesis. It is the only logical operation which introduces any new idea; for induction does nothing but determine a value, and deduction merely evolves the necessary consequences of a pure hypothesis. Deduction proves that something must be; Induction shows that something actually is operative; Abduction merely suggests that something may be. Its only justification is that from its suggestion deduction can draw a prediction which can be tested by induction, and that, if we are ever to learn anything or to understand phenomena at all, it must be by abduction that this is to be brought about.“ (C.P 5.171).

8) Unsere Forschungsfrage legt es nahe, insbesondere das Konzept des Spurenlesers von Gerhard Hard (1995) zu studieren, das wir hier allerdings nicht diskutieren können. Wer mit der SemiotikDiskussion vertraut ist, wird feststellen, dass wir unsere eigen Überlegungen am semiotischen Pragmatismus ausrichten, während Hard sich überwiegend an der französischen Semiologie orientiert.

\section{Literatur}

Balsinger, P.W., 1996: Überlegungen und Bemerkungen hinsichtlich einer Methodologie interdisziplinärer Wissenschaftspraxis. In: Balsinger, P.W.; Defila, R.; Di Giulio, A. (Hrsg.): Ökologie und Interdisziplinarität - eine Beziehung mit Zukunft? Wissenschaftsforschung zur Verbesserung der fachübergreifenden Zusammenarbeit. Basel u.a.: Birkhäuser, S. 73-85

Bergmann, M., 2003: Indikatoren für eine diskursive Evaluation transdisziplinärer Forschung. In: Technikfolgenabschätzung - Theorie und Praxis, Heft 1, 12. Jg., S. 65-75

Brandes, D.; Griese, D., 1991: Siedlungs- und Ruderalvegetation von Niedersachsen. Eine kritische Übersicht. Braunschweig: Universitätsbibliothek der Technischen Universität Braunschweig

Büchi, H., 1996: Das Paradoxe mit der Transdisziplinarität. In: GAIA 5/5, S. 205-208

Dechent, H.-J., 1988: Wandel der Dorfflora. Münster-Hiltrup: KTBL-Schriften-Vertrieb im Landwirtschaftsverlag $\mathrm{GmbH}$
Dilthey, $W ., 1924$ : Ideen über eine beschreibende und zergliedernde Psychologie [1894]. In: Dilthey, W.: Gesammelte Werke Bd. 5. Leipzig, Berlin: Teubner, S. $139-240$

Eco, U.; Seboek, T.A., 1983: The Sign of Three. Dupin, Holmes, Peirce. Bloomington, Ind.: Indiana University Press

Foucault, M., 1980: Power - Knowledge. Selected Interviews and other Writings, 1972-1977. New York: Pantheon Books

Gibbons, M.; Limoges, C.; Nowotny, H.; Schwartzmann, S.; Scott, P.; Trow, M., 1994: The New Production of Knowledge. The Dynamics of Science and Research in Contemporary Societies. London: Sage

Ginzburg, C., 1979: Spie. Radici di un paradigma indiziarion. In: A. Gargani (ed.): Crisis della ragione. Turin: Einaudi, S. 57-106

Grunwald, A., 1999: Transdisziplinäre Umweltforschung: Methodische Probleme der Qualitätssicherung. In: TA-Datenbank-Nachrichten, Heft 3/4, 8. Jg., S. 32-39

Grunwald, A., 2002: Nachhaltigkeitsforschung in der Helmholtz-Gemeinschaft. GAIA 11/1, S. 31-33

Hard, G., 1995: Spuren und Spurenleser. Zur Theorie und Ästhetik des Spurenlesens in der Vegetation und anderswo. Osnabrück: Rasch

Henkel, G., 1999: Der Ländliche Raum. Gegenwart und Wandlungsprozesse seit dem 19. Jahrhundert in Deutschland. Stuttgart, Leipzig: Teubner

Hof, H., 2002: Umweltforschung im fächerübergreifenden Verbund. Probleme von Forschung und Forschungsförderung und ein aktuelles Förderangebot. In: GAIA 11/1, S. 26-28

Horkheimer, M., 1967: Zur Kritik der instrumentellen Vernunft. Frankfurt/Main: Fischer

Husserl, E., 1982: Die Krisis der europäischen Wissenschaften und die transzendentale Phänomenologie. Eine Einleitung in die phänomenologische Philosophie. Hamburg: Meiner

Jaeger, J.; Scheringer, M., 1998: Transdisziplinarität: Problemorientierung ohne Methodenzwang. In: GAIA 7/1, S. 10-25

Lienenbecker, H.; Raabe, U., 1993: Die Dorfflora Westfalens. Bielefeld: Naturwissenschaftlicher Verlag für Bielefeld und Umgebung

Ludwig, W., 1972: Chenopodium botrys, Ch. schraderanum und $\mathrm{Ch}$. pumilio (= Bestimmungsarbeiten in Botanischen Gärten N.F.10). Hessische Floristische Briefe 21, S. 2-6

Mittelstraß, J., 1992: Auf dem Wege zur Transdisziplinarität. In: GAIA 1/5, S. 250

Otte, A.; Ludwig, T., 1990: Planungsindikator dörfliche Ruderalvegetation - ein Beitrag zur Fachpla 
nung Grünordnung/Dorfökologie. Teil 1: Methode zur Kartierung und Bewertung. München: Bayerisches Staatsministerium für Ernährung, Landwirtschaft und Forsten

Peirce, C.S., 1966: Lectures on Pragmatism. C.P. Peirce, Collected Papers Volume V. Cambridge, Mass.: The Belknap Press of Harvard University Press, S. 13-131, hier: S. 106

Peirce, C.S., 1983: Phänomen und Logik der Zeichen. Frankfurt am Main: Suhrkamp

Peirce, C.S., 1985: Die Festigung der Überzeugung und andere Schriften. Frankfurt a.M.: Ullstein

Scheringer, M., 2002: Institutionelle Ansätze zur Förderung fächerübergreifender Umweltforschung. In: GAIA 11/1, S. 25

Snow, C.P., 2001: The two cultures. Reprint, Cambridge: Cambridge University Press

Tretter, F., 1997: Zur Institutionalisierung der Transdisziplinarität. In: GAIA 6/3, S. 167-168

Wächter, M., 2002: Der Förderschwerpunkt „Sozial-ökologische Forschung" im BMBF. In: GAIA 11/1, S. 28-31

Weingart, $P ., 1997$ : Interdisziplinarität - der paradoxe Diskurs. In: Ethik und Sozialwissenschaften 8/4, S. 521-529

\section{Kontakt}

Dr. Stefan Brunzel

Dr. Jens Jetzkowitz

AG Gesellschaft und Ökologie

Philipps-Universität Marburg

Karl-von-Frisch-Straße 8 A, 35032 Marburg

Tel.: + 49 (0) $6421 / 282$ - 4316 oder -4315

Fax: + 49 (0) $6421 / 282-8978$

E-Mail: brunzel@staff.uni-marburg.de

Internet:

$$
\text { jetzkowi@staff.uni-marburg.de }
$$

http://www.fogoe.de/forschungsprojekte.htm 